(Aus der kgl. ung. tierphysiol. Versuchsstation in Budapest.

Vorstand: Univ.-Prof. Franz Tangl.)

\title{
Versuche über die Verdaulichkeit des Chitins und den Nährwert der Insecten.
}

Von

\section{Di. Arthur Zaitschek.}

Über die Verdaulichkeit des Chitins und über den Nährwert der Insecten stehen uns bisher nur die Untersuchungen von E. Wolff, W. Funke und G. Dittmann ${ }^{1}$ ) zur Verfügung. Sie stellten die Verdaulichkeit des Maikäfers beim Schweine fest, indem sie mit einem Gemenge von Gersteschrot und Maikäfern Ausnützungsversuche anstellten. Die Maikäfer wurden durch heisses Wasser getödtet, auf einer Malzdarre getrocknet und mittelst einer Kartoffelreibe zerrieben. Im Ganzen führten sie bei drei Schweinen sechs Einzelversuche mit dem Gemenge von Gerste und Maikäfern aus, wobei sich für den Maikäfer folgende Verdauungscoëfficienten ergaben:

\begin{tabular}{c|c|c|c|c}
\hline \hline & $\begin{array}{c}\text { Organische } \\
\text { Substanz }\end{array}$ & $\begin{array}{c}\text { Gesammt- } \\
\text { Stickstoff }\end{array}$ & $\begin{array}{c}\text { Protein- } \\
\text { substanz }\end{array}$ & Fett \\
\hline 1 & 54,73 & 58,72 & 64,90 & 79,15 \\
2 & 54,74 & 60,56 & 66,91 & 80,76 \\
3 & 50,35 & 56,29 & 62,36 & 78,83 \\
4 & 59,45 & 66,31 & 73,27 & 83,20 \\
5 & 59,01 & 64,86 & 71,68 & 84,94 \\
6 & 64,21 & 67,59 & 74,67 & 91,33 \\
\hline Im Mittel : & 57,08 & 62,39 & 68,97 & 83,04
\end{tabular}

Das Chitin erwies sich in allen Versuchen als unverdaulich. Die Trockensubstanz der frisch getödteten Maikäfer hatte die folgende Zusammensetzung:

Proteïn Fett Chitin Extractstoffe Reinasche Sand

$\begin{array}{llllll}66,65 & 12,05 & 16,06 & 0,57 & 4,52 & 0,18 .\end{array}$

1) Versuche über das Verdaungsvermögen der Schweine für verschiedene Futtermittel und Futtermischungen. Die landw. Versuchestationen Bd. 19 S. 241. 
Vers. üb. die Verdaulichkeit des Chitins u. den Nährwert der Insecten. 613

Da dieser einzelstehende Versuch noch der Ergänzung bedarf, begrüsste ich mit Freude die Gelegenheit, den Nährwert einer anderen Insectenart, der sogenannten Theissblüte (Uferaas, Palingenia longicauda Oliv.) feststellen zu können. Die Theissblüte ist eine gemeine Art der Familie der Eintagsfliegen (Ephemeridae), welche in die Ordnung der Pseudoneuroptera gehört.

Das Uferaas steigt aus der Theiss ${ }^{1}$ ) zwischen 10. und 20. Juni in riesigen Massen empor, welche Erscheinung das Blühen der Theiss genannt wird. Das Schwärmen dieser Insecten beginnt Nachmittags um 5 Uhr und hört zugleich mit dem Leben dieser Insecten schon um 8 Uhr Abends auf, worauf der Strom. ihre verwesten Körper in grossen Mengen fortschwemmt. Diese Leichen werden dann in Kähnen in geeigneter Weise gesammelt, auf Matten ausgebreitet, getrocknet und als Düngemittel, oder als Geflügel- bezw. Fischfutter, in Form von Klössen auch als Fischlockspeise verwendet ${ }^{1}$ ).

Es ist meine Pflicht, zu erwähnen, dass ich diese entomologischen Daten Herrn Josef Lósy, Assistenten an der biesigen entomologischen Versuchsstation, verdanke.

Mit Rücksicht auf die Verwendung der Theissblüte als Geflügelfutter habe ich den Nährwert dieses Insectes am Geflügel in zwei Versuchen bestimmt; das Geflügel nahm das Uferaas ohne jedes Beifutter auf. In einem Versuch A wurde die Theissblüte allein, in einem zweiten Versuch B mit Gerste verfüttert. Die zu diesen Versuchen notwendige Menge von Theissblüte hat uns die kgl. ung. entomologische Versuchsstation, welche auch die Sammlung des Materials besorgte, gefälligst zur Verfügung gestellt.

Die Zusammensetzung der Theissblüte bestimmte ich nach den bei der Futtermittelanalyse gebräuchlichen Methoden; nur wurde statt der Rohfaser, welche im tierischen Organismus nicht vorkommt, das Chitin bestimmt. Es wird übrigens in derselben Weise bestimmt wie die Rohfaser nạch der Weender Methode ${ }^{2}$ ).

Mehrere Proben (à $2 \mathrm{~g}$ ) der feingepulverten Theissblüte wurden mit verdünnter Säure und Lauge, dann mit Wasser gekocht, mit Alkohol und Äther gewaschen und in Porcellan-Goochtiegelchen

1) Auch an der Elbe sollen Ephemeriden in grösseren Schwärmen vorkommen, die dann durch grosse, an den Ufern angelegte Feuer angelockt werden. Die mit verbrannten Flügeln niederfallenden Insecten sammelt man und bringt sie-unter dem Namen "Weisswurm" als Vogel- oder Fischfutter in den Handel.

2) Hoppe-Seyler, Thierfelder's Chem. Analyse S. 208.

E. Pflügør, Archiv für Plysiologie. Bd. 104. 
durch Asbest filtriert. Der Rückstand wurde bei $110^{\circ}$ C. bis zur Gewichtsconstanz getrocknet und gewogen. Fünf Bestimmungen gaben die folgenden Resultate:

$$
\begin{aligned}
& \text { 1. . . . . } 0,2294 \\
& \text { 2. . . . . } 0,2280 \\
& \text { 3. . . . . . } 0,2259 \\
& \text { 4. . . . . } 0,2250 \\
& \text { 5. . . . . . } 0,2266 \\
& \text { Im Mittel: . . 0,2270. }
\end{aligned}
$$

In einem Teil des Chitin-Rückstandes bestimmte ich den Aschegehalt, welchen ich im Mittel $0,0646 \mathrm{~g}$ fand, so dass der aschefreie Teil $0,1624 \mathrm{~g}$ beträgt. In anderen Proben bestimmte ich den $\mathrm{N}$-Gehalt, welcher $0,0122 \mathrm{~g}$ ausmachte. Da nach der Formel $\mathrm{C}_{18} \mathrm{H}_{30} \mathrm{~N}_{2} \mathrm{O}_{12}$ in $0,1624 \mathrm{~g}$ Chitin nur $6,34 \%$, d. i. $0,0103 \mathrm{~g} \mathrm{~N}$ enthalten sind, so dürfte die Differenz zwischen dem gefundenen und dem berechneten $\mathrm{N}: 0,0122 \mathrm{~g}-0,0103 \mathrm{~g}=0,0019 \mathrm{~g} \mathrm{~N}$ aus den ungelöst gebliebenen Eiweissstoffen stammen, weshalb dieser $\mathrm{N}$ mit 6,25 multipliciert $[(0,0019 \times 6,25)=0,0121 \mathrm{~g}$ Eiweiss $]$ vom aschefreien Rückstand abgezogen wurde. Es verbleibt also auf diese Weise $0,1624-0,0121=0,1503 \mathrm{~g}$ reinen, asche- und eiweissfreien Chitins, d. i. $7,53 \%$. Ich wollte durch Pepsin-Salzsäure-Verdaung aus dem Chitin das Eiweiss entfernen. Dies gelang mir aber nur zum kleinen Teil; denn der N-Gehalt hat sich dadurch nur wenig' verringert. Dies findet seine Erklärung darin, dass es unmöglich war, den Chitin-Rückstand zur Verdauung fein zu pulverisieren; ausserdem dürfte das langandauernde Trocknen bei hoher Temperatur die Eiweissstoffe derart verändert haben, dass deren Verdaulicbkeit stark abnahm.

Natürlich musste sowohl bei Berechnung der N-haltigen Stoffe aus lem nach Kjeldahl bestimmten $\mathrm{N}$ wie auch bei Bestimmung des Reinproteïngehaltes nach Bernste.in ${ }^{1}$ ) der N-Gehalt des Chitins berücksichtigt werden. So z. B. wurde aus dem $10,58 \%$ betragenden Gesammt- $\mathrm{N}$ der Theissblute die dem Chitin entsprechenden $0,45 \%$ abgezogen, und nur der Rest, $10,13 \%$, wurde mit 6,25 multipliciert. Gerade so verfuhren wir bei Berechnung des Reimproteingehaltes. Auf diese Weise ergibt sich die folgende Zusammensetzung der Theissblüte:

1) Landw. Versuchsstationen Bd. 54 S. 327. 
Vers. äb. die Verdaulichkeit des Chitins u. den Nährwert der Insecten. 615

\begin{tabular}{|c|c|c|}
\hline & $\begin{array}{l}\text { Zusammensetzung der } \\
\text { lufttrockenen } \\
\text { Theisblüte }\end{array}$ & $\begin{array}{c}\text { Zusammensetzung der } \\
\text { Theissblüte- } \\
\text { Trockensubstanz }\end{array}$ \\
\hline 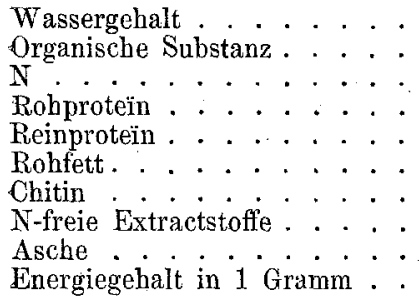 & $\begin{array}{r}12,42 \% \\
79,01 \% \\
10,58 \% \\
63,31 \% \\
58,87 \% \\
4,38 \% \\
7,13 \% \\
4,19 \% \\
8,57 \% \\
4568 \mathrm{cal} \%\end{array}$ & $\begin{array}{c}0 \\
90,21 \% \\
12,08 \% \\
72,29 \% \\
67,22 \% \\
5,00 \% \\
8,14 \% \\
4,78 \% \\
9,79 \% \\
5216 \mathrm{cal}\end{array}$ \\
\hline
\end{tabular}

Aus dieser Zusammensetzung verdient die grosse Menge der N-haltigen Substanzen und der hohe calorische Wert hervorgehoben zu werden. (Letzterer wurde mit der Berthelot-Mahler'schen Bombe bestimmt.)

Zum Versuch wurden 15 Stück Hühner in einem geräumigen, zu Stoffwechselversuchen dienenden Käfig eingestellt, der annähernd ebenso gross ist, wie die bei uns zur Hühnermästung verwendeten Steigen. Der Boden dieses ganz zerlegbaren Stoffwechselkastens ist mit Zinkblech bedeckt, und ca. $12 \mathrm{~cm}$ über dem Boden befinden sich verzinnte Eisenstäbe in nicht zu engen Intervallen, auf welchen die Tiere sitzen, so dass sich die Excremente am Boden des Kastens sammeln, wo sie dann in einem geschlossenen Gefäss verwahrt werden. Die verzinnten Eisenstäbe können aus dem Kasten herausgenommen werden, so dass nach Beendigung eines Versuches die am Boden und an den Stäben anhaftenden Excremente gut abgewaschen werden können. Die solcherart gewonnenen Waschwässer werden natürlich eingedampft und den Excrementen hinzugefügt. Das Futter und das Wasser wird dem Geflügel in Gefässen, welche vor dem Kasten angebracht sind, gereicht.

Im Versuch A gewöhnten wir die 15 Stück Hühner ungarischer Landrasse allmählich an die Theissblüte, indem diesen früher mit Mais gefütterten Tieren allmählich mehr und mehr Mais entzogen and mit Uferaas ersetzt wurde, bis sie am Ende nur dieses Futter erhielten. Dieses nahmen sie im Anfange widerwillig auf; später aber gewöhnten sie sich so daran, dass ein Huhn durehschnittlich täglich $60 \mathrm{~g}$ verzehrte. Nach Beginn der Fütterung mit Theissblüte kamen vier Hühner um, so dass dieser Versuch A nur mit elf Stück Hühnern - 21 Tage hindurch - ausgeführt wurde.

Den Verlauf des Versuches, welcher sich auf die Bestimmung der Ausnutzung des Rohfettes und des Chitins sowie des $\mathrm{N}$ - und 
Energieumsatzes erstreckte, zeigt weiter unten das Versuchsprotokoll A. Die Bestimmung der Ausnutzung musste auf das Rohfett und Chitin beschränkt werden, da die Ausnutzung des Eiweisses beim Geflügel nur nach Anlage eines Anus praeternaturalis festzustellen ist. Da wir unsere Versuche gleichzeitig an einer grösseren Anzahl von Hühnern und unter möglichst natürlichen Verhältnissen anstellen wollten, haben wir auf diesen operativen Eingriff verzichtet:

\section{Versuch A.}

Vorfutterung vom 1.-6. October 1903. Dauer des Versuches vom 6. bis 27. October 1903. Futter der elf Hühner während des Versuches $13998 \mathrm{~g}$ Theissblüte. Futter pro Tag $666,6 \mathrm{~g}$ Theissblüte. Futter pro Tag und Huhn 60,6 g Theissblüte.

\begin{tabular}{|c|c|c|c|c|c|}
\hline \multirow{3}{*}{$\begin{array}{l}\text { Nummer } \\
\text { des } \\
\text { Huhnes }\end{array}$} & \multicolumn{5}{|c|}{ Körpergewicht am } \\
\hline & 6. & 14. & 17. & 23. & 27. \\
\hline & \multicolumn{5}{|c|}{ October 1903 in Gramm } \\
\hline $\begin{array}{l}21 \\
23 \\
24 \\
25 \\
26 \\
27 \\
28 \\
30 \\
31 \\
32 \\
34\end{array}$ & $\begin{array}{l}692 \\
605 \\
670 \\
772 \\
462 \\
500 \\
670 \\
602 \\
712 \\
565 \\
570\end{array}$ & $\begin{array}{l}748 \\
632 \\
660 \\
805 \\
500 \\
525 \\
702 \\
652 \\
757 \\
555 \\
635\end{array}$ & $\begin{array}{l}858 \\
770 \\
742 \\
890 \\
600 \\
590 \\
790 \\
808 \\
870 \\
642 \\
780\end{array}$ & $\begin{array}{l}890 \\
840 \\
770 \\
915 \\
630 \\
652 \\
655 \\
905 \\
955 \\
710 \\
850\end{array}$ & $\begin{array}{l}937 \\
727 \\
757 \\
930 \\
632 \\
672 \\
682 \\
925 \\
952 \\
727 \\
814\end{array}$ \\
\hline $\begin{array}{l}\text { Zusammen . . } \\
\text { Im Durchschn. pro Huhn }\end{array}$ & $\begin{array}{r}6820 \\
620\end{array}$ & $\begin{array}{r}7171 \\
652\end{array}$ & $\begin{array}{r}8340 \\
758\end{array}$ & $\begin{array}{r}8772 \\
797\end{array}$ & $\begin{array}{r}8755 \\
796\end{array}$ \\
\hline Versuchstag & $\begin{array}{c}\text { Excremente } \\
\mathrm{g}\end{array}$ & & Versuchstag & & $\begin{array}{l}\text { Excremente } \\
\mathrm{g}\end{array}$ \\
\hline $\begin{array}{r}1 \\
2 \\
3 \\
4 \\
5 \\
6 \\
7 \\
8 \\
9 \\
10\end{array}$ & $\begin{array}{l}1640 \\
1790 \\
1852 \\
1786 \\
2030 \\
2070 \\
2040 \\
2850 \\
2675 \\
2905\end{array}$ & & $\begin{array}{l}11 \\
12 \\
13 \\
14 \\
15 \\
16 \\
17 \\
18 \\
19 \\
20 \\
21\end{array}$ & & $\begin{array}{l}2980 \\
2530 \\
2905 \\
2540 \\
2670 \\
2620 \\
2485 \\
2490 \\
2945 \\
2580 \\
2700\end{array}$ \\
\hline $\begin{array}{l}\text { Zusammen } \\
\text { Im Durchschnitt pro } \\
\text { Im Durchschnitt pro }\end{array}$ & ho & & $\begin{array}{l}\therefore . \\
. \\
.\end{array}$ & & $\begin{array}{r}50583 \\
2408 \\
219\end{array}$ \\
\hline
\end{tabular}


Vers. üb. die Verdaulichkeit des Chitins u. den Nährwert der Insecten. 617

Die Zusammensetzung der verfütterten Theissblüte war schon auf S. $613 \mathrm{f}$. mitgeteilt.

In den Excrementen wurde das Chitin in derselben Weise wie in der Theissblüte bestimmt. Die $\mathrm{Zus}$ a m mensetzung der frischen Excremente war die folgende:

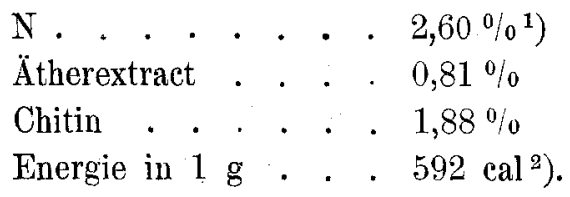

Die Ausnutzung pro Tag und Huhn war die folgende: Ätherextract Chitin

In den Excrementen entleert . . 2,59 g 4,21 g Im Futter aufgenommen . . . 1,78 " 4,12, Resorbiert . . . . . . 0,81 " 0,09 " $" \quad$ in Procenten . . . 31,5 "2,1

N-Umsatz pro Tag und Huhn:

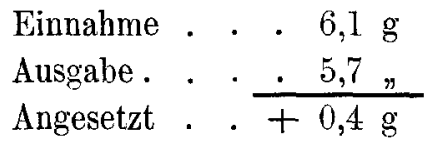

$=$ eine Fleischbildung von ca. $12 \mathrm{~g}$ pro Tag und Huhn.

Energieumsatz pro Tag und Huhn:

Einnahme: Theissblute (a) . . 270,1 Cal.

Ausgaben: Excremente . . . . 129,5 ,

Dazu kommen für angesetzten $\mathrm{N}$

Summe der Ausgaben (b) . . . . 139,3 Cal.

Physiol. nutzbare Energie (a-b) . 130,8 "

Nach diesem Versuche sind $48,4 \%$ der Energie der Theiss. blüte physiol, nutzbar. Auf die eingehendere Besprechung der Versuchsergebnisse kommen wir noch zurück.

Nach Beendigung des Versuches überzeugten wir uns davon, dass das Füttern des Uferaases dem Fleisch der Hühner einen sehr unangenehmen Geruch verleiht. Wohl verschwindet dieser. Geruch

1) In der lufttrockenen Substanz nach Kjeldahl bestimmt, sowobl in diesen wie in den übrigen Versuchen.

2) Der Energiegehalt der Excremente wurde in diesem wie in den Versuchen $B$ und $C$ durch Verbrennung der lufttrockenen Substanz in der Berthelot-Mahler'schen Bombe bestimmt. Zur Verhütung des N-Verlustes beim Trocknen wurde den frischen Excrementen Weinsäure in genau abgewogener Menge zugesetzt und deren Energie dann vom gefundenen Brennwert in Abzug gebracht. 
beim Braten oder Kochen grösstenteils, immerhin behält aber das Fleisch der nur mit Uferaas gefütterten Hühner einell unangenehmen Geruch und Geschmack.

Der Versuch B, der als Controlle des Versuches A diente, hatte auch den Zweck, darüber Aufklärung zu geben, ob der unangenehme Gesehmack des Huhnfleisches auch dann bemerkbar ist, wenn das Uferaas einem anderen Futtermittel zugesetzt wird. In diesem Versuche wurde die Theissblüte mit Gerste verfüttert.

Den Verlauf und die Ergebnisse dieses Versuches zeigen die folgenden Tabellen:

\section{Versuch B.}

Vorfütterung vom 25. November bis 1. December 1903. Dauer des Versuches vom 1.-15. December 1903. Futter der vier Hühner während des Versuches $2100 \mathrm{~g}$ Gerste $+2100 \mathrm{~g}$ Theissblüte. Futter pro Tag $150 \mathrm{~g}$ Gerste + $150 \mathrm{~g}$ Theissblüte. Futter pro Tag und Huhn $37,5 \mathrm{~g}$ Gerste $+37,5 \mathrm{~g}$ Theissblüte.

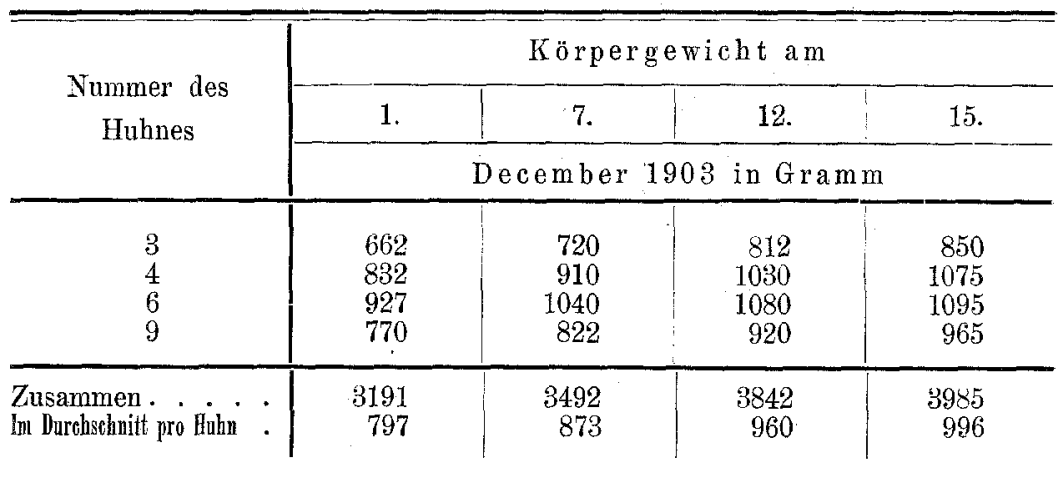

\begin{tabular}{c|c|c|c}
\hline Versuchstag & $\begin{array}{c}\text { Excremente } \\
\mathrm{g}\end{array}$ & Versuchstag & $\begin{array}{c}\text { Excremente } \\
\mathrm{g}\end{array}$ \\
\hline & 530 & 8 & 665 \\
2 & 492 & 9 & 740 \\
3 & 600 & 10 & 715 \\
4 & 480 & 11 & 695 \\
5 & 645 & 12 & 775 \\
6 & 550 & 13 & 740 \\
7 & 705 & 14 & 700 \\
\hline
\end{tabular}

Zusammen

Im Durchschnitt pro Tag.

9032

Im Durchschnitt pro Tag und Huhn

645

161,2

Zusammensetzung des Futters.

Theissblüte: Wassergehalt . . . . 12,16\% (siehe die $\mathrm{Zu}$ Gerste: Wassergehalt . . . $13,94 \%$ sammensetzung Asche . . . . . . $3,29 \%$ S. 615 ). 
Vers. üb. die Verdaulichkeit des Chitins u. den Nährwert der Insecten. 619

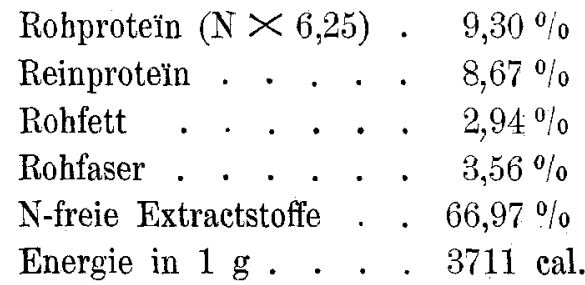

Zusammensetzung der frischen Excremente:

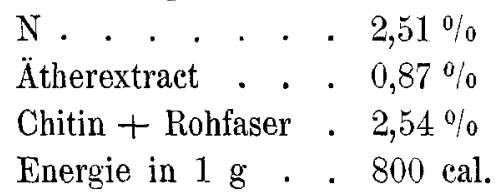

Ausnutzung:

Ätherextract

In der Theissblüte aufgenommen . $1,65 \mathrm{~g} \quad 2,68 \mathrm{~g}$ Chitin

" "Gerste " 1,10 n 1,35 "Rohfaser

Zusammen aufgenommen . . . $2,75 \mathrm{~g} \quad 4,03 \mathrm{~g} \mathrm{Ch}+\mathrm{R}$.

In den Excrementen entleert . $\cdot 1,40,4,10, \mathrm{Ch} .+\mathrm{R}$.

Resorbiert . . . . . . . . $1,35 \mathrm{~g} \quad 0$

$"$

in Procenten . . . . 49,1, 0

$\mathrm{N}-\mathrm{Umsatz}$ :

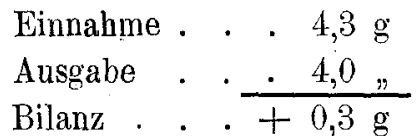

Energie-Umsatz:

Einnahme: Theissblüte . . . . . 171,8 Cal.

Gerste . . . . . 139,2

Summa der Einnahmen (a) . . 311,0 Cal.

Ausgaben: Excremente . . . . 129,5 "

Dazu kommen für angesetzten $\mathrm{N}$

Summe der Ausgaben (b)

$\frac{6,1 \mathrm{n}}{135,6 \mathrm{Cal} \text {. }}$

Physiol. verwertbare Energie (a-b) 175,4 Cal.

Der Energieumsatz zejgt eine bedeutend bessere Verwertung, indem von der Energie des Gerste-Uferaasgemenges $56,4 \%$ physiol. verwertbar sind. Der Geruch des Uferaases war im Fleisch und Fett dieser Hühner bedeutend schwächer, aber immerhin noch wahrnehmbar.

Um auch aus Versuch B den Nährwert des Uferaases berechnen zu können, war es notwendig, in einem besonderen Versuch C den Nährwert der mit dem Uferaas verfütterten Gerste für sich zu bestimmen. Wir haben also gleichzeitig mit Versuch $B$ vier Hühner derselben 
Rasse in einen besonderen Käfig eingestellt und 13 Tage hindureh mit der im Versuch $B$ verwendeten Gerste gefüttert. Der Verlauf und die Ergebnisse des Versuches $\mathrm{C}$ sind die folgenden:

\section{Versuch C.}

Verfütterung vom 25. November bis 1. December 1903. Dauer des Versuches rom 1.-12. December 1903. Futter der vier Hühner während des Versuches $2100 \mathrm{~g}$ Gerste. Futter pro Tag $290 \mathrm{~g}$ Gerste. Futter pro Tag und Huhn $72,5 \mathrm{~g}$ Gerste.

\begin{tabular}{c|c|c|c}
\hline \multirow{2}{*}{$\begin{array}{c}\text { Nummer des } \\
\text { Huhnes }\end{array}$} & \multicolumn{3}{|c}{ Körpergewicht a m } \\
& 1. & 7. & 12. \\
\hline & \multicolumn{3}{|c|}{ December 1903 in Gram m } \\
\hline 5 & 724 & 782 & 830 \\
7 & 802 & 815 & 732 \\
8 & 929 & 975 & 990 \\
10 & 589 & 615 & 640 \\
\hline Zusammen . . . . . & 3044 & 3187 & 3192 \\
Im Durchschn. pro Huhn & 760 & 794 & 798
\end{tabular}

\begin{tabular}{c|c|c|c}
\hline Versuchstag & $\begin{array}{c}\text { Excremente } \\
\mathrm{g}\end{array}$ & Versuchstag & $\begin{array}{c}\text { Excremente } \\
\mathrm{g}\end{array}$ \\
\hline & 425 & 6 & 375 \\
2 & 420 & 7 & 350 \\
3 & 440 & 8 & 392 \\
4 & 440 & 9 & 380 \\
5 & 422 & 10 & 310 \\
& & 11 & 302 \\
\hline Zusammen . . . . . . . . . . . . . . . . . . . & 4256 \\
Im Durchschnitt pro Tag . . . . . . . . . . . . . . & 387 \\
Im Durchschnitt pro Tag und Hubn . . . . . . . . . . & 96,7 \\
\end{tabular}

Zusammensetzung der Excremente:

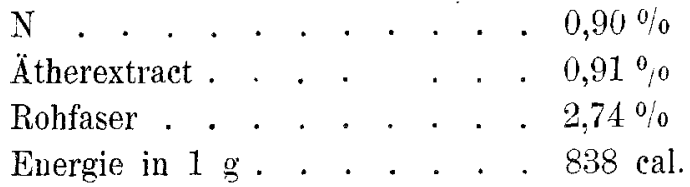

Ausnutzung:

Ätherextract Rohfaser

Im Futter aufgenommen . . . . 2,14 g 2,62 g

In den Excrementen entleert . . 0,88 " 2,65 "

Resorbiert . . . . . . . . 1,26, 0

" in Procenten . . . 58,9, 0 
Vers. üb. die Verdaulichkeit des Chitins u. den Nährwert der Insecten.

$\mathrm{N}-\mathrm{U}$ msatz:

Einnahmen . . $\quad 1,1 \mathrm{~g}$
Ausgaben . · $\cdot 0,9 n$
Bilanz . . . $\cdot+0,2 \mathrm{~g}$

Energie-Umsatz:

Einnabmen: Gerste (a) . . . . 269,5 Cal.

Ausgaben: Excremente . . . . . 81,1 "

Dazu kommen für angesetzten $\mathrm{N}$.

Summe der Ausgaben (b)

$\begin{array}{r}5,6 \quad n \\ \hline \quad 86,7 \mathrm{~g} \\ \hline 182,8 \mathrm{~g}\end{array}$

Der Energieumsatz in Versuch $\mathrm{C}$ zeigt, dass im Huhn $67,8 \%$ der Energie der Gerste physiologisch verwertbar sind.

Es sei gleich an dieser Stelle, da ich später nicht darauf zurückkomme, darauf hingewiesen, dass dieser Versuch, wie bereits von anderen und auch uns ${ }^{1}$ ) und neuerdings von Lehmann ${ }^{2}$ ) gefunden wurde, die vollkommene Unverdaulichkeit der Rohfaser im Geflügel bestätigt.

Mit Hilfe dieses Versuches C können wir num berechnen, wieviel von dem in Versuch B resorbierten Fett und von der verwertbaren Energie auf die Gerste entfällt; der Rest kommt dem Uferaas zu. Wie die folgende Berechnung ergibt, zeigen die Resultate der Versuche $\mathrm{A}$ und $\mathrm{B}$ bezüglich der Ausnutzung des Fettes eine genügende, die Unverdaulichkeit des Chitins und die verwertbare Energie (den physiologischen Nutzeffect) betreffend eine vollkommene Übereinstimmung.

Die Ausnutzung des Fettes der Theissblüte in Versuch B lässt sich folgendermaassen berechnen:

Resorbiert wurden insgesammt Fett (siehe

S. 619). . . . . . . . . . . $1,35 \mathrm{~g}$

Hiervon entfallen (nach Versuch C) auf

die Gerste . . . . . . . . 0,65"

Es bleiben also für die Theissblüte . . 0,70 "Fett $=42,4 \%$ der mit der Theissblüte verzehrten $1,65 \mathrm{~g}$ Fett.

Da nach Versuch A 31,5\%, nach Versuch B, wie eben berechnet, $42,4 \%$ des Theissblütenfettes resorbierbar sind, so erhält man im

1) Pflüger's Arch. Bd. 93 S. 98.

2) Nach einem Referat in Biedermann's Centralbl. f. Agrikulturchemie Jahrg. 33 H. 6 S. 417. 
Mittel beider Versuche $37,0 \%$. Nach diesem Mittelwerte sind in $100 \mathrm{~g}$ Trockensubstanz der Theissblüte $1,85 \mathrm{~g}$ im Huhn resorbierbares Rohfett enthalten. Vergleichshalber sei erwähnt, dass in $100 \mathrm{~g}$ Gerstetrockensubstanz 2,00 g, in $100 \mathrm{~g}$ Maistrockensubstanz hingegen bedeutend mehr, nämlich $4,9 \mathrm{~g}$, im Huhn verdauliches Rohfett enthalten ist.

Vom verfütterten Chitin fehlen in den Excrementen im Versuch A $2 \%$, im Versuch B nichts. Daraus können wir ohne Weiteres folgern, dass das Chitin in den Hühnern ganz unverdaulich ist; denn die $2 \%$ fallen weit innerhalb der Versuchsfehler. (Im Versuch B wurde im Kote noch etwas mehr Chitin gefunden.) Das Chitin, die Stützsubstanz der niederen Tiere, verhält sich also im Huhn ganz genau so wie die Cellulose.

Aus dem Energieumsatz in dem Versuche B lässt sich auf Grund des Umsatzes in Versuch $\mathrm{C}$ die Verwertung der chemisehen Energie der Theissblüte, wie folgt, berechnen:

Physiol. verwertbar sind nach Versucb B insgesammt 175,4 Cal. Hiervon entfallen (nach Versuch C) auf die Gerste 94,4 " Also bleiben für die Theissblüte . . . . . . 81,0 " d. i. $=47,2 \%$, da mit der Theissblüte 171,8 Calorien Energie aufgenommen wurden: Bei dieser Berechnung musste noch angenommen werden, was ja auch zulässig ist, dass der im Körper angesetzte $\mathrm{N}$ - (in Versuch B) - zu gleichen Teilen aus der Gerste und der Theissblüte stammt.

Da von der chemischen Energie der Theissblüte

nach Versuch A . . . 48,4\%

nách Versuch B . . . 47,2\% physiol. verwertbar sind so ergibt sich im Mittel $47,8 \%$

als "relativer" (Tangl) physiologischer Nutzeffect (Rubner's „physiologischer Nutzeffect") der Theissblüte beim Huhn.

Wenn $47,8 \%$ der chemischen Energie der Theissblüte im Huhn verwertet werden, so entbält

$1 \mathrm{~g}$ Trockensubstanz der Theissblüte . 2493 cal.

1 " organischer Substanz der Theissblüte 2764 "

im Huhn physiologiseh verwertbare chemische Energie. (Diesen auf die Gewiehtseinheit bezogenen Gehalt an verwertbarer chemischer Energie nennt Tangl den "specifischen physiologisehen Nutzeffect".) 
Vers. üb. die Verdaulichkeit des Chitins u. den Nährwert der Insecten. 623

Vergleichshalber habe ich im Folgenden die auf den Gehalt an (für das Huhn) verwertbarer Energie (physiologischem Nutzeffect) bezüglichen Zahlen der Theissblüte und der Gerste neben einander gestellt :

Es entbält:

$$
\begin{aligned}
& \text { in der Theissblitte in der Gerste } \\
& \text { physiologisch nutzbare Energie }
\end{aligned}
$$

1 g Substanz (bei 13,94\% Wasser-

$$
\text { gehalt) }^{1} \text { ) . . . . . . . } 2146 \text { cal } 2516 \text { cal }
$$

$1 \mathrm{~g}$ Trockensubstanz . . . . . 2493 " 2924 "

$1 \mathrm{~g}$, organischer Substanz . . . $2764 " 3040 "$

relativer physiologischer Nutzeffect $\quad 47,2 \% \quad 67,8 \%$.

Die chemische Energie der Gerste wird also im Huhn besser verwertet wie die der Theissblüte; $100 \mathrm{~g}$ Theissblüte sind etwa $85,3 \mathrm{~g}$ Gerste gleichwertig.

Die Untersuchungen wurden auf Anregung und unter der Leitung des Herrn Prof. F. Tangl ausgeführt.

1) Auf den Wassergehalt der Gerste umgerechnet, da nur bei gleichem Wassergehalt ein Vergleich zulässig ist. 